\title{
Range identification for nonlinear parameterizable paracatadioptric systems ${ }^{\text {k }}$
}

\author{
Nitendra Nath ${ }^{\mathrm{a}, *}$, Enver Tatlicioglu ${ }^{\mathrm{b}}$, Darren M. Dawson ${ }^{\mathrm{a}}$ \\ a Department of Electrical \&' Computer Engineering, Clemson University, Clemson, SC 29634-0915, United States \\ ${ }^{\mathrm{b}}$ Department of Electrical \& Electronics Engineering, Izmir Institute of Technology, Urla, Izmir, 35430, Turkey
}

\section{A R T I C L E I N F O}

\section{Article history:}

Received 20 July 2009

Received in revised form

5 January 2010

Accepted 15 March 2010

Available online 20 April 2010

\section{Keywords:}

Nonlinear parameterization

Range identification

Paracatadioptric systems

Lyapunov methods

Vision-based estimation

min-max algorithm

\begin{abstract}
A B S T R A C T
In this paper, a new range identification technique for a calibrated paracatadioptric system mounted on a moving platform is developed to recover the range information and the three-dimensional (3D) Euclidean coordinates of a static object feature. The position of the moving platform is assumed to be measurable. To identify the unknown range, first, a function of the projected pixel coordinates is related to the unknown 3D Euclidean coordinates of an object feature. This function is nonlinearly parameterized (i.e., the unknown parameters appear nonlinearly in the parameterized model). An adaptive estimator based on a min-max algorithm is then designed to estimate the unknown 3D Euclidean coordinates of an object feature relative to a fixed reference frame which facilitates the identification of range. A Lyapunov-type stability analysis is used to show that the developed estimator provides an estimation of the unknown parameters within a desired precision. Numerical simulation results are presented to illustrate the effectiveness of the proposed range estimation technique.
\end{abstract}

(c) 2010 Elsevier Ltd. All rights reserved.

\section{Introduction}

The problem of range identification, the estimation of the unknown time-varying distance of the object from the camera along its optical axis, has received noteworthy attention over the last several years due to its significance in several applications such as autonomous vehicle navigation, aerial tracking, path planning, surveillance, etc. These applications require either the range or the 3D Euclidean coordinates of features of a moving or a static object to be recovered from their two-dimensional (2D) image sequence. The range estimation is usually done by mounting a camera on a moving vehicle such as a mobile robot or an unmanned aerial vehicle (UAV) which captures images of the static objects or features. However, the use of conventional (perspective) cameras poses restrictions for some applications because of their limited field-of-view (FOV).

One efficient way to enhance the FOV is to use mirrors (spherical, elliptical, hyperboloid, or paraboloid) in conjunction with conventional cameras, commonly known as catadioptric

\footnotetext{
is This work is supported in part by a DOE Contract, and a Honda Corporation Grant. The material in this paper was partially presented at the IEEE International Conference on Systems, Man, and Cybernetics, October 11-14, 2009, San Antonio, TX USA. This paper was recommended for publication in revised form by Associate Editor Shuzhi Sam Ge under the direction of Editor Miroslav Krstic.

* Corresponding author. Tel.: +1 864656 7218; fax: +1 8646567220.

E-mail addresses: nnath@clemson.edu (N. Nath), enver@envertatlicioglu.com (E. Tatlicioglu), darren.dawson@ces.clemson.edu (D.M. Dawson).
}

systems (Baker \& Nayar, 1999). However, the use of curved mirrors reduces the resolution and distorts the images to a large extent. As stated in Hu, Aiken, Gupta, and Dixon (2008), the distorted image mapping can be dealt with by using computer vision techniques, but the nonlinearity which is introduced in the transformation makes it difficult to recover the $3 \mathrm{D}$ coordinates of the object features. Catadioptric systems that have a single effective viewpoint are known as central catadioptric systems, and are desirable because they allow a distortion-free reconstruction of panoramic images (Orghidan, Mouaddib, \& Salvi, 2005). A paracatadioptric system is a special case of central catadioptric systems which employs a paraboloid mirror along with an orthographic lens. These systems are advantageous due to the fact that the paraboloid constant of the mirror and its physical size do not need to be determined during the calibration. Furthermore, mirror alignment requirements are relaxed, so the mirror can be arbitrarily translated enabling the camera to zoom in on a part of the paraboloid mirror for higher resolution; however, with a reduced FOV (Baker \& Nayar, 1999).

In the past, many researchers have proposed various range identification techniques for perspective vision systems. Some of these have utilized the extended Kalman filter (EKF) (Chiuso, Favaro, Jin, \& Soatto, 2002; Kano, Ghosh, \& Kanai, 2001; Matthies, Kanade, \& Szeliski, 1989). However, EKF involves linearization of the nonlinear vision model and requires a priori knowledge of the noise distribution. To overcome the shortcomings of the linear model, many researchers focused on utilizing nonlinear system analysis and estimation tools to develop nonlinear observers to 
identify the range when the motion parameters were known (Chen \& Kano, 2002, 2004; Dixon, Fang, Dawson, \& Flynn, 2003; Jankovic \& Ghosh, 1995; Karagiannis \& Astolfi, 2005; Ma, Chen, \& Moore, 2004). More recently, in Nath, Braganza, and Dawson (2008a), the measurement of camera position was utilized to develop an adaptive estimator to recover the structure; this was extended in Nath, Braganza, Dawson, and Burg (2008b) to recover the range.

Although there have been several reports on range identification for perspective vision systems, very few results have been shown for range identification for catadioptric systems. Ma, Chen, and Moore (2005) proposed a range identification technique for paracatadioptric system based on a sequence of linear approximation-based observers. Gupta, Aiken, $\mathrm{Hu}$, and Dixon (2006) designed a nonlinear observer to asymptotically identify the range for a paracatadioptric system. However, both of these reports assumed the focal point of the paraboloid mirror to be at its vertex. This assumption was recently relaxed in Hu et al. (2008). In the current work, we also base our development on a more practical approach that the focus of the paraboloid mirror is not at its vertex. In Orghidan et al. (2005), an omnidirectional light projector was embedded in a paracatadioptric system, and the range was calculated by triangulation. Hu et al. (2008) developed a nonlinear estimator similar to Dixon et al. (2003) to identify the range for paracatadioptric systems where the motion parameters were assumed to be known, and it assumed that the object must translate in at least one direction.

In this paper, we present a method to identify the range of a static object using a moving paracatadioptric system whose position is measurable. For many applications, position measurements are considerably less noisy than velocity measurements; hence, we are motivated to develop an estimator based on position measurements. The estimator is designed by first developing a geometric model along with a paracatadioptric projection model that relates an object feature with the paracatadioptric system mounted on a moving mechanical system. The novelty of this work lies in the compensation for nonlinear parameterization of the model which relates the projected pixel coordinates to the Euclidean coordinates of the object feature. It should be noted that contrary to Nath et al. (2008a), where the unknown terms appear linearly in the parameterized model for a perspective vision system, in the current work, the unknown parameters appear nonlinearly in the model for a paracatadioptric system. This fact makes it difficult to use a standard adaptive estimator or a gradient based estimator (Annaswamy, Skantze, \& Loh, 1998). The estimator presented in this paper which facilitates range identification to the desired precision is based on a min-max optimization algorithm. We show that the developed estimator identifies the range and the 3D coordinates of the object feature upon the satisfaction of a Nonlinear Persistent Excitation (NLPE) condition and is robust to noise, as demonstrated by the simulation results. The contributions of this paper are that: (i) the developed estimator utilizes position measurements instead of velocity measurements, (ii) is continuous, and (iii) provides estimation of unknown parameters within a desired precision. A preliminary version of this paper has appeared in Nath, Tatlicioglu, and Dawson (2009a).

\section{Model development}

\subsection{Geometric model}

For the development of a geometric relationship between a moving paracatadioptric system and a stationary object, an orthogonal coordinate frame, denoted by $\mathcal{M}$, which is centered at the focal point of the moving paraboloid mirror whose $Z$-axis is aligned with the optical axis of the camera, is defined (see Fig. 1). As shown in Fig. 1, an inertial coordinate frame, denoted by $\mathcal{W}$,

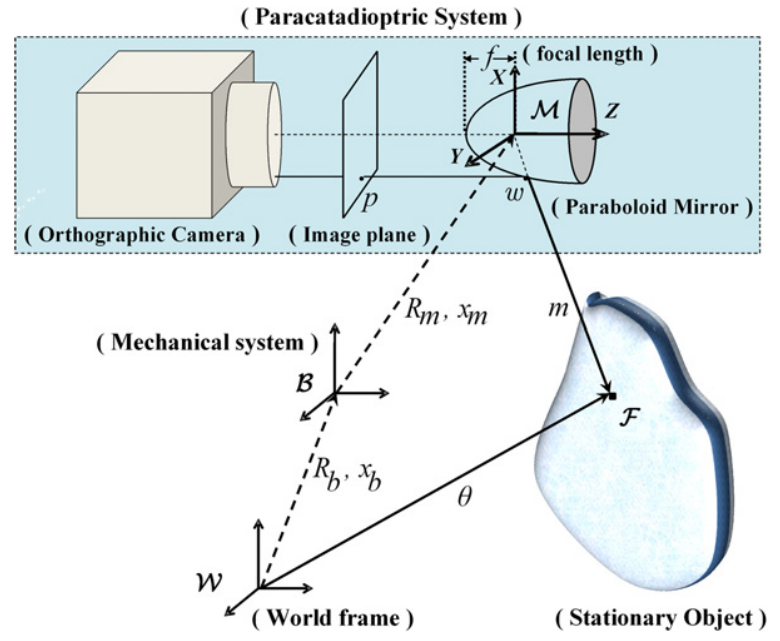

Fig. 1. Geometric relationships between the stationary object, mechanical system, and the paracatadioptric system.

and an orthogonal coordinate frame, denoted by $\mathscr{B}$, are defined. $\mathcal{F}$ denotes a static feature on a stationary object. Let the unknown 3D Euclidean coordinates of the object feature be denoted as the constant $\theta \in \mathbb{R}^{3}$ relative to the world frame $\mathcal{W}$ and $m(t) \in \mathbb{R}^{3}$ relative to $\mathcal{M}$ be defined as follows

$m \triangleq\left[\begin{array}{lll}x & y & z\end{array}\right]^{T}$.

To relate the coordinate systems, let $R_{b}(t) \in S O(3)$ and $x_{b}(t) \in \mathbb{R}^{3}$ denote the measurable rotation matrix and the translation vector, respectively, from $\mathscr{B}$ to $\mathcal{W}$ expressed in $\mathcal{W}$. Let $R_{m} \in S O(3)$ and $x_{m} \in \mathbb{R}^{3}$ be the known rotation matrix and the translation vector, respectively, from $\mathcal{M}$ to $\mathscr{B}$ expressed in $\mathcal{B}$.

\subsection{Paracatadioptric system projection model}

In a paracatadioptric system, a Euclidean point is projected onto a paraboloid mirror and is then reflected to an orthographic camera (see Fig. 1); thus, to facilitate the subsequent development, and to relate the geometric model to the vision system, let the projection of the object feature on the surface of the paraboloid mirror with its focus at the origin be denoted by $w(t) \in \mathbb{R}^{3}$ relative to $\mathcal{M}$ and defined as follows

$w \triangleq\left[\begin{array}{lll}u & v & q\end{array}\right]^{T}$.

The projection $w(t)$ can be expressed as follows (Geyer \& Daniilidis, 2000)

$w=\frac{2 f}{\lambda} m=\frac{2 f}{\lambda}\left[\begin{array}{lll}x & y & z\end{array}\right]^{T}$

where $f \in \mathbb{R}$ is the known focal length of the mirror and $\lambda(x, y, z)$ $\in \mathbb{R}$ is the unknown nonlinear signal defined as follows

$\lambda \triangleq-z+\sqrt{x^{2}+y^{2}+z^{2}}$.

It is worthwhile to mention that the use of a paracatadioptric system results in an orthographic projection from the paraboloid mirror to the image plane. In other words, the reflected rays are parallel to the optical axis; thus, the distance from the mirror to the image plane is irrelevant. After utilizing (2) and (3), the projection can be expressed as follows

$\left[\begin{array}{l}u \\ v\end{array}\right]=\frac{2 f}{\lambda}\left[\begin{array}{l}x \\ y\end{array}\right]$. 


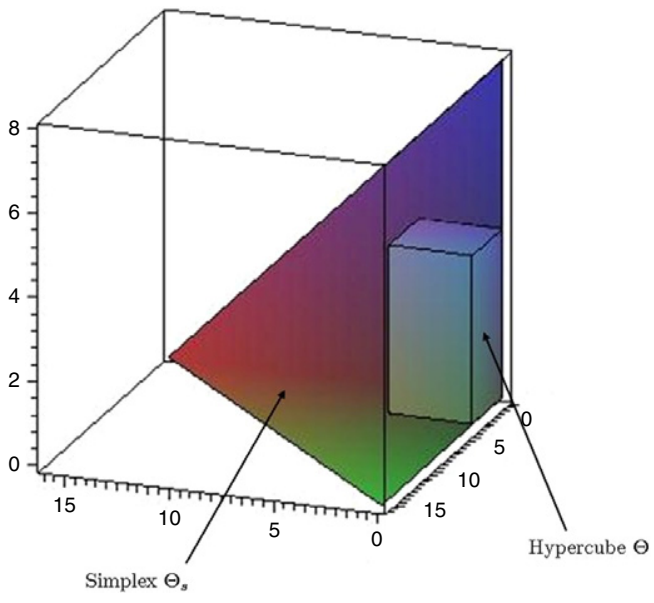

Fig. 2. Simplex $\Theta_{s}$, and hypercube $\Theta$.

However, when measured from a CCD chip as in any practical case, $[u, v]^{T}$ is transformed as follows (Geyer \& Daniilidis, 2002)

$p \triangleq\left[\begin{array}{l}u^{\prime} \\ v^{\prime}\end{array}\right]=K\left[\begin{array}{l}u \\ v\end{array}\right]+C$

where $p(t) \in \mathbb{R}^{2}$ are the measured pixel coordinates on the image plane, $K \in \mathbb{R}^{2 \times 2}$ and $C \in \mathbb{R}^{2}$ are defined as follows

$K \triangleq\left[\begin{array}{ll}a_{1} & a_{2} \\ 0 & a_{1}^{-1}\end{array}\right] \quad C \triangleq\left[\begin{array}{l}c_{x} \\ c_{y}\end{array}\right]$

where $a_{1}^{2}, a_{2} \in \mathbb{R}$ are the aspect ratio and the skew factor, respectively, and $C$ is the image center. Since a central catadioptric camera can be calibrated using a single image of three lines (Barreto \& Araujo, 2005; Geyer \& Daniilidis, 2002), we assume the camera to be calibrated. It is clear from (6) that the coordinates in the mirror frame, $u(t)$ and $v(t)$, can be obtained from the measured pixel coordinates as follows

$\left[\begin{array}{ll}u & v\end{array}\right]^{T}=K^{-1}(p-C)$.

Also, since the paraboloid mirror is rotationally symmetric, $q(\cdot) \in$ $\mathbb{R}$ can be computed from $u(t)$ and $v(t)$ as follows (Hu et al., 2008)

$q=\frac{u^{2}+v^{2}}{4 f}-f$.

Assumption 1. It is assumed that the object feature is not on the optical axis i.e., $x(t), y(t) \neq 0$ simultaneously and thus, $\lambda(\cdot) \neq 0$.

\section{Nonlinear parameterization of the model}

In this section, the parameterization of the nonlinear function $q(\cdot)$ is presented after relating it to the unknown 3D Euclidean coordinates of the object feature. From Fig. 1, $m(t)$ can be written as follows (Nath et al., 2008a)

$m=R_{m}^{T}\left[R_{b}^{T}\left(\theta-x_{b}\right)-x_{m}\right]$.

After utilizing (1), the 3D coordinates of the object feature relative to $\mathcal{M}$ can be expressed as follows

$x=R_{m 1}^{T}\left[R_{b}^{T}\left(\theta-x_{b}\right)-x_{m}\right]$

$y=R_{m 2}^{T}\left[R_{b}^{T}\left(\theta-x_{b}\right)-x_{m}\right]$

$z=R_{m 3}^{T}\left[R_{b}^{T}\left(\theta-x_{b}\right)-x_{m}\right]$ where $R_{m i}^{T} \in \mathbb{R}^{1 \times 3}$ is the $i$ th row of $R_{m}^{T}$, and $z(t)$ is the range of the object feature. After substituting (5) into the nonlinear model given in (9), $q(\cdot)$ is nonlinearly parameterized (NLP) as follows

$q\left(\theta, R_{m}, x_{m}, R_{b}, x_{b}, f\right)=\frac{\left(\frac{2 f}{\lambda} x\right)^{2}+\left(\frac{2 f}{\lambda} y\right)^{2}}{4 f}-f$.

In the subsequent analysis, $q\left(\theta, R_{m}, x_{m}, R_{b}, x_{b}, f\right)$ is replaced by $q(\theta, \Pi)$, where $\Pi(\cdot) \in \mathbb{R}^{n_{1} \times n_{2}}$, with $n_{1}$ and $n_{2}$ being integers, contains the combinations of known and measurable quantities (i.e., $f, R_{m}, x_{m}, R_{b}(t)$, and $x_{b}(t)$ ).

Remark 1. It can be seen from (4), (9) and (11)-(13) that $\theta$ appears nonlinearly in $q(\cdot)$; thus, it is not possible to give an exact expression for the function $\Pi(\cdot)$. Specifically, $\Pi(\cdot)$ is a shorthand notation for $\Pi\left(f, R_{m}, x_{m}, R_{b}(t), x_{b}(t)\right)$.

Assumption 2. The unknown parameter vector $\theta$ is assumed to belong to a known hypercube $\Theta \subset \mathbb{R}^{3}$. In other words, the 3D coordinates of the object feature relative to $W$ are assumed to lie within their known minimum and maximum values.

Assumption 3. For any $\Pi(\cdot)$, the function $q(\cdot)$ is either concave or convex on a simplex ${ }^{1} \Theta_{s}$ in $\mathbb{R}^{3}$ such that $\Theta_{s} \supset \Theta$ (see Fig. 2).

Assumption 4. The function $\Pi(t)$ is a bounded, continuous function of its arguments, and is Lipschitz in $t$ such that

$\left\|\Pi\left(t_{1}\right)-\Pi\left(t_{2}\right)\right\| \leq L_{1}\left|t_{1}-t_{2}\right| \quad \forall t_{1}, t_{2} \in \mathbb{R}^{+}$

where $L_{1} \in \mathbb{R}^{+}$is the Lipschitz constant.

Assumption 5. $q\left(\theta_{0}, \Pi\right)$ is Lipschitz with respect to its arguments such that

$\left|q\left(\theta_{0}+\Delta \theta_{0}, \Pi+\Delta \Pi\right)-q\left(\theta_{0}, \Pi\right)\right| \leq L_{2}\left(\|\Delta \Pi\|+\left\|\Delta \theta_{0}\right\|\right)$

where $L_{2} \in \mathbb{R}^{+}$is the Lipschitz constant, $\Delta \Pi=\Pi\left(t_{1}\right)-\Pi\left(t_{2}\right)$, and $\Delta \theta_{0}=\theta_{0}\left(t_{1}\right)-\theta_{0}\left(t_{2}\right)$.

Definition 1. A function $H(\varsigma)$ is said to be convex on $\Theta$ if it satisfies the following inequality

$$
\begin{aligned}
H\left(\sigma \varsigma_{1}+(1-\sigma) \varsigma_{2}\right) \leq & \sigma H\left(\varsigma_{1}\right)+(1-\sigma) H\left(\varsigma_{2}\right) \\
& \forall \varsigma_{1}, \varsigma_{2} \in \Theta
\end{aligned}
$$

and concave if it satisfies the following inequality

$$
\begin{aligned}
H\left(\sigma \varsigma_{1}+(1-\sigma) \varsigma_{2}\right) \geq & \sigma H\left(\varsigma_{1}\right)+(1-\sigma) H\left(\varsigma_{2}\right) \\
& \forall \varsigma_{1}, \varsigma_{2} \in \Theta
\end{aligned}
$$

where $0 \leq \sigma \leq 1$.

Remark 2. It should be noted that the Assumptions 2 and 3 essentially characterize the nature of the nonlinear parameterization, and the convexity or concavity of the function $q(\cdot)$ is required in a region $\Theta_{s}$ which is larger than the hypercube $\Theta$. Also, since an estimation problem for a practical moving paracatadioptric system is considered in this paper, we assume that the measurable position signals and its velocity are bounded. The boundedness of the position signals in other words mean that the function $\Pi(\cdot)$ is bounded.

Remark 3. In Appendix C, we show how Assumptions 4 and 5 can be replaced by a simpler condition on the differentiability of $q(\cdot)$ for the estimation problem.

\footnotetext{
1 A simplex in $\mathbb{R}^{n}$ is a convex polyhedron having exactly $n+1$ vertices.
} 
Remark 4. Assumptions 4 and 5 are related to the boundedness of the motion of the mechatronic platform (i.e., a robot manipulator, UAV, or a mobile robot, etc.) to which the omnidirectional camera is attached. Also, the definition of convexity and concavity are provided in Definition 1. As noted in Boyd and Vandenberghe (2004), for an affine function, we always have equality in (17) and (18), respectively. Therefore all affine (and therefore also linear) functions are both convex and concave. Conversely, any function that is convex and concave is affine. In Assumption 3, it is claimed that for any $\Pi(\cdot), q(\cdot)$ is either convex or concave. Thus, Assumption 3 is utilized to exclude affine (and also linear) functions.

Remark 5. The hypercube $\Theta$ can be found using the minimum and the maximum values of $\theta$. The vertices of the simplex $\Theta_{s}$, denoted by $\theta_{s 1}, \theta_{s 2}, \theta_{s 3}, \theta_{s 4} \in \mathbb{R}^{3}$, can be found by first inscribing $\Theta$ in a 3-dimensional sphere and then inscribing this sphere inside a 4-dimensional polyhedron (Annaswamy et al., 1998; Sommerville, 1958)

It should be noted that in (11)-(13), $\theta$ (i.e, the constant $3 \mathrm{D}$ coordinates of the object feature relative to $W$ ) is the only unknown vector, and if we estimate this we can then obtain an estimation of the 3D coordinates of the object feature relative to $\mathcal{M}$ as follows

$\hat{x}=R_{m 1}^{T}\left[R_{b}^{T}\left(\hat{\theta}-x_{b}\right)-x_{m}\right]$

$\hat{y}=R_{m 2}^{T}\left[R_{b}^{T}\left(\hat{\theta}-x_{b}\right)-x_{m}\right]$

$\hat{z}=R_{m 3}^{T}\left[R_{b}^{T}\left(\hat{\theta}-x_{b}\right)-x_{m}\right]$

where $\hat{x}(t), \hat{y}(t) \in \mathbb{R}$ are the estimates of $x(t)$ and $y(t)$, respectively, $\hat{z}(t) \in \mathbb{R}$ is the estimate of the corresponding range $z(t)$, and $\hat{\theta}(t) \in \mathbb{R}^{3}$ is the estimate of $\theta$.

Remark 6. It is worthwhile to mention that the range identification precision depends on the precision to which the constant unknown parameter vector $\theta$ is estimated. The estimated range, $\hat{z}(t)$, further depends upon the noise in the measurable position signals $R_{b}(t)$ and $x_{b}(t)$, and the error in the constant camera calibration parameters $R_{m}$ and $x_{m}$. The effect of noise in these signals will be demonstrated later in the simulation section.

\section{Range estimation}

In this section, an estimator is presented for the unknown constant parameter vector $\theta$ which appears nonlinearly in the model given in (14). There are very few researchers who have addressed adaptive control or estimation for NLP systems (Annaswamy et al., 1998; Boskovic, 1996; Fomin, Fradkov, \& Yakubovich, 1981; Ortega, 1998). Parameter convergence in NLP systems was addressed in Cao, Annaswamy, and Kojic (2003). As pointed out in Annaswamy et al. (1998), the gradient algorithm employed in Boskovic (1996), Fomin et al. (1981) and Ortega (1998) are not only inadequate but can also lead to instability for general NLP systems. In this work, we design an adaptive estimator that facilitates the identification of range within a desired precision based on the min-max algorithm developed in Annaswamy et al. (1998). The maximization is that of a tuning function over all the possible values of the nonlinear parameters, and the minimization is over all the possible sensitivity functions that can be used in the adaptive law. A sensitivity function, which differs from the gradient depending upon the sign of a tuning error, is incorporated in the adaptive law. The stability analysis ensures that the use of the tuning function, along with the adaptive law, has globally bounded error signals, and upon the satisfaction of an NLPE condition similar to Cao et al. (2003), the parameter estimation follows; hence, the identification of range.

\subsection{Estimator design}

To facilitate the estimator design, the estimate of (14) is defined as follows

$\hat{q} \triangleq \frac{\left(\frac{2 f}{\hat{\lambda}} \hat{x}\right)^{2}+\left(\frac{2 f}{\hat{\lambda}} \hat{y}\right)^{2}}{4 f}-f$

where $\hat{q}(\cdot) \in \mathbb{R}$ denotes $q(\hat{\theta}), \hat{\lambda}(\hat{x}, \hat{y}, \hat{z}) \in \mathbb{R}$ is the estimate of $\lambda(\cdot)$ and is defined as follows

$\hat{\lambda} \triangleq-\hat{z}+\sqrt{\hat{x}^{2}+\hat{y}^{2}+\hat{z}^{2}}$.

To further facilitate the development, we define a filter signal $q_{f}(t) \in \mathbb{R}$ as follows

$\dot{q}_{f} \triangleq-\alpha q_{f}+q ; \quad q_{f}(0) \triangleq 0$

where $\alpha \in \mathbb{R}^{+}$. The estimate of (24) is designed as follows

$\dot{\hat{q}}_{f}=-\alpha\left(\hat{q}_{f}-\varepsilon \operatorname{sat}(r)\right)+\hat{q}-a^{*} \operatorname{sat}(r)$

where $\hat{q}_{f}(t), \dot{\hat{q}}_{f}(t) \in \mathbb{R}$ are the estimates of $q_{f}(t)$, and $\dot{q}_{f}(t)$, respectively, $\varepsilon \in \mathbb{R}^{+}$is the desired precision, $a^{*}(t)$ is the tuning function obtained from the subsequently presented min-max optimization problem, and $r(t) \in \mathbb{R}$ is defined as follows

$r \triangleq \frac{\tilde{q}_{f}}{\varepsilon}$

where the filter error $\tilde{q}_{f}(t) \in \mathbb{R}$ is defined as follows

$\tilde{q}_{f} \triangleq \hat{q}_{f}-q_{f}$.

Also, in (25), sat $(r)$ is a saturation function given as follows

$\operatorname{sat}(r)= \begin{cases}+1 & \text { if } r \geq 1 \\ r & \text { if }|r|<1 \\ -1 & \text { if } r \leq-1\end{cases}$

To proceed with the development, we define a tuning error $\tilde{q}_{f \varepsilon}(t) \in$ $\mathbb{R}$ as follows

$\tilde{q}_{f \varepsilon} \triangleq \tilde{q}_{f}-\varepsilon \operatorname{sat}(r)$.

After taking the time derivative of (27), the following expression can be written

$\dot{\tilde{q}}_{f}=-\alpha \tilde{q}_{f \varepsilon}+\hat{q}-q-a^{*} \operatorname{sat}(r)$

where (24), (25) and (29) were utilized.

Remark 7. It should be noted that the inclusion of the tuning error $\tilde{q}_{f \varepsilon}(t)$ provides the following expressions

$\tilde{q}_{f \varepsilon}=0 \quad$ when $\left|\tilde{q}_{f}\right| \leq \varepsilon$

$\dot{\tilde{q}}_{f \varepsilon}=\dot{\tilde{q}}_{f} \quad$ when $\left|\tilde{q}_{f}\right|>\varepsilon$.

This remark is utilized later in the stability analysis.

Based on the stability analysis an estimator $\dot{\hat{\theta}}(t) \in \mathbb{R}^{3}$ is designed with a projection strategy which facilitates the estimation of $\theta$ as follows

$$
\dot{\hat{\theta}}=\operatorname{Proj}\left\{-\tilde{q}_{f \varepsilon} \phi^{*}\right\}
$$

where $\phi^{*}(t) \in \mathbb{R}^{3}$ is the sensitivity function. The projection strategy $\operatorname{Proj}\{\cdot\}$ in (31) ensures that $\hat{\theta}(t)$ always belongs to the hypercube $\Theta$. The strategy is as follows

$\hat{\theta}_{j}= \begin{cases}\hat{\theta}_{j} & \text { if } \hat{\theta}_{j} \in\left[\theta_{j, \min }, \theta_{j, \max }\right] \\ \theta_{j, \min } & \text { if } \hat{\theta}_{j}<\theta_{j, \min } \\ \theta_{j, \max } & \text { if } \hat{\theta}_{j}>\theta_{j, \max }\end{cases}$ 
where the subscript $j$ denotes the $j$ th element of the corresponding vector $\forall j=1,2,3$, and $\theta_{j, \min }, \theta_{j, \max } \in \mathbb{R}$ are the minimum and maximum values of the $j$ th component of $\theta$, respectively.

Similar to Annaswamy et al. (1998), the solutions for $\phi^{*}(t)$ and $a^{*}(t)$ are obtained from a min-max optimization problem of the following form

$a^{*}=\min _{\phi \in \mathbb{R}^{3}} \max _{\theta \in \Theta_{s}} J(\phi, \theta)$

$\phi^{*}=\arg \min _{\phi \in \mathbb{R}^{3}} \max _{\theta \in \Theta_{s}} J(\phi, \theta)$

where the performance index $J(\cdot) \in \mathbb{R}$ is given by the following expression

$J(\cdot)=\operatorname{sat}(r)\left[\hat{q}-q-\tilde{\theta}^{T} \phi\right]$

where $\tilde{\theta}(t) \in \mathbb{R}^{3}$ is the parameter estimation error defined as follows

$\tilde{\theta} \triangleq \hat{\theta}-\theta$.

The solutions of (33) and (34) are given as follows ${ }^{2}$

(a) when $\tilde{q}_{f}<0$

$a^{*}= \begin{cases}0 & \text { if } q \text { is concave on } \Theta_{s} \\ A_{1} & \text { if } q \text { is convex on } \Theta_{s}\end{cases}$

$\phi^{*}= \begin{cases}\nabla q(\hat{\theta}) & \text { if } q \text { is concave on } \Theta_{s} \\ A_{2} & \text { if } q \text { is convex on } \Theta_{s} .\end{cases}$

(b) when $\tilde{q}_{f} \geq 0$

$a^{*}= \begin{cases}A_{1} & \text { if } q \text { is concave on } \Theta_{s} \\ 0 & \text { if } q \text { is convex on } \Theta_{s}\end{cases}$

$\phi^{*}= \begin{cases}A_{2} & \text { if } q \text { is concave on } \Theta_{s} \\ \nabla q(\hat{\theta}) & \text { if } q \text { is convex on } \Theta_{s} .\end{cases}$

In (37)-(40), $A(t) \in \mathbb{R}^{4}$ is given as follows

$A=\left[A_{1} A_{2}\right]^{T}=G^{-1} b$

where $A_{1}(t) \in \mathbb{R}$, and $A_{2}(t) \in \mathbb{R}^{3}, G(t) \in \mathbb{R}^{4 \times 4}$ is given as follows

$G=\left[\begin{array}{cc}-1 & \beta\left(\hat{\theta}-\theta_{s 1}\right)^{T} \\ -1 & \beta\left(\hat{\theta}-\theta_{s 2}\right)^{T} \\ -1 & \beta\left(\hat{\theta}-\theta_{s 3}\right)^{T} \\ -1 & \beta\left(\hat{\theta}-\theta_{s 4}\right)^{T}\end{array}\right]$

and $b(t) \in \mathbb{R}^{4}$ is given as follows

$b=\left[\begin{array}{l}\beta\left(\hat{q}-q_{s 1}\right) \\ \beta\left(\hat{q}-q_{s 2}\right) \\ \beta\left(\hat{q}-q_{s 3}\right) \\ \beta\left(\hat{q}-q_{s 4}\right)\end{array}\right]$

where $\beta(\Pi) \in \mathbb{R}$ is defined as follows

$\beta= \begin{cases}1 & \text { if } q \text { is convex on } \Theta_{s} \\ -1 & \text { if } q \text { is concave on } \Theta_{s} .\end{cases}$

In (43), $g_{s h} \triangleq q\left(\theta_{s h}, \Pi\right) \forall h=1,2,3,4$. As mentioned earlier in Remark 5, $\theta_{\text {sh }}$ are the vertices of the simplex $\Theta_{s}$. In (38) and (40), $\nabla q(\hat{\theta}) \in \mathbb{R}^{3}$ is the gradient function given as follows

$\nabla q(\hat{\theta})=\left.(\partial q / \partial \theta)\right|_{\theta=\hat{\theta}}$.

It is evident that the estimate of the constant 3D coordinates of the object feature relative to the world frame $($ i.e., $\hat{\theta}(t))$ can be used to obtain the estimates of all its 3D coordinates relative to the vision system, including the range (i.e., $\hat{z}(t))$ from (19)-(21).

2 The reader is referred to Annaswamy et al. (1998) for the proof of the solutions
Remark 8. It should be noted that the inclusion of the tuning error $\tilde{q}_{f \varepsilon}(t)$ with the saturation function $\operatorname{sat}(r)$ ensures that the estimator is continuous, even if a discontinuous solution of the min-max algorithm is obtained (see Annaswamy et al., 1998 for more detailed description).

Remark 9. It should be noted that $\hat{\theta}(t)$ is bounded because of the projection strategy in (32); thus, $\phi^{*}(t)$ can be upper bounded as follows

$\left\|\phi^{*}(t)\right\| \leq L_{\phi} \quad \forall t \geq t_{0}$

where $L_{\phi} \in \mathbb{R}^{+}$.

Remark 10. We note that the tuning function $a(t)$, the sensitivity function, and the matrices $G(t)$ and $b(t)$ are similar to the corresponding functions defined in Annaswamy et al. (1998) and Cao et al. (2003). The novelty in the proposed work lies in the fact that we have applied a modified version of Annaswamy et al. (1998) and Cao et al. (2003) for nonlinear parameter estimation application (i.e., range identification using paracatadioptic systems). The work presented in Annaswamy et al. (1998) deals with the control of a nonlinearly parameterized system, while parameter convergence of a nonlinearly parameterized system is presented in Cao et al. (2003). In the current paper, we modify the technique given in Cao et al. (2003) for nonlinearly parameterized parameter estimation, which facilitates the range identification for a paracatadioptric system. This modification is accomplished through the introduction of a filtering scheme which is given in (24).

\subsection{Stability analysis}

Theorem 1. The adaptive update law given in (31) along with the solutions of $a^{*}(t)$ and $\phi^{*}(t)$ given in (37)-(40) ensures that $\tilde{q}_{f \varepsilon}(t) \in$ $\mathcal{L}_{2} \cap \mathcal{L}_{\infty}$; hence, the stability of the estimator, and the global boundedness of the overall adaptive system are ensured.

Proof. See Appendix A.

Theorem 2. The developed estimation technique ensures that $\|\tilde{\theta}(t)\|$ $\leq \sqrt{\gamma}$ as $t \rightarrow \infty$ provided the following NLPE condition holds

$\beta\left(\Pi\left(t_{2}\right)\right)\left(q\left(\hat{\theta}\left(t_{1}\right), \Pi\left(t_{2}\right)\right)-q\left(\theta, \Pi\left(t_{2}\right)\right)\right) \geq \varepsilon_{u}\left\|\hat{\theta}\left(t_{1}\right)-\theta\right\|$

where

$\gamma=\frac{8 \varepsilon c_{1}}{\varepsilon_{u}^{2}} ; \quad c_{1}=4 L_{1} L_{2}+2 L_{2} L_{\phi}+L_{\phi}^{2}$,

$t_{2} \in\left[t_{1}, t_{1}+T_{0}\right], t_{1}>t_{0}$, and $T_{0}, \varepsilon_{u} \in \mathbb{R}^{+}$.

Proof. See Appendix B.

Remark 11. From the definition of $\gamma$ in (48), it follows that $\gamma$ can be made smaller by choosing smaller $\varepsilon$. As the desired precision $\varepsilon \rightarrow 0$, then $\gamma \rightarrow 0$; thus, the parameter estimation error $\|\tilde{\theta}(t)\| \rightarrow 0$.

Remark 12. As pointed out in Cao et al. (2003), it is difficult to check if the NLPE condition given in (47) can be satisfied in a general nonlinear system. To ensure parameter convergence, $\Pi(\cdot)$ must be such that one of the following occurs at least at one time instant $t_{2} \in\left[t_{1}, t_{1}+T\right]$ : (a) For the given $\tilde{\theta}(t), \Pi(\cdot)$ must change in such a way that the sign of $\hat{q}(\cdot)-q(\cdot)$ is reversed, while keeping the convexity/concavity of $q(\cdot)$ the same or, (b) for the given $\tilde{\theta}(t), \Pi(\cdot)$, must reverse the convexity/concavity of $q(\cdot)$, while preserving the sign of $\hat{q}(\cdot)-q(\cdot)$. The reader is referred to Cao et al. (2003) for a detailed analysis. It should be noted that the parameter convergence shown in the subsequently presented simulation results seems to indicate that the NLPE condition for the particular problem attacked in this paper was met. 


\section{Simulation results}

A detailed simulation study was conducted to evaluate the performance of the proposed estimation technique using the Mathworks Simulink program. The translation vector $x_{b}(t)$, and angular rotation $q_{b}(t) \in \mathbb{R}^{3}$ (yaw-pitch-roll about $x-y-z$ axes) to the mechanical system were given as follows

$x_{b}=\left[\begin{array}{llll}\sin (\pi t) & 2 \cos (\pi t) & \sin (2 \pi t)\end{array}\right]^{T} \quad[\mathrm{~m}]$

$q_{b}=\left[\begin{array}{lll}0 & 0 & 0.2 \cos (\pi t)\end{array}\right]^{T} \quad[\mathrm{rad}]$.

The measurable signal $R_{b}(t)$ was generated using $q_{b}(t)$. The 3D Euclidean coordinates of an object feature relative to the world frame, $\theta$, were taken as follows

$\theta=\left[\begin{array}{lll}1 & 2 & 2\end{array}\right]^{T} \quad[\mathrm{~m}]$

along with the following maximum and minimum values

$\theta_{\max }=\left[\begin{array}{lll}4 & 4 & 4\end{array}\right]^{T} \quad[\mathrm{~m}] ; \quad \theta_{\min }=\left[\begin{array}{lll}0 & 0 & 0\end{array}\right]^{T} \quad[\mathrm{~m}]$.

The calibration parameters were set as $a_{1}=1, a_{2}=0$, and $C=\left[\begin{array}{ll}0 & 0\end{array}\right]^{T}$. The rotation matrix $R_{m}$, and the translation vector $x_{m}$ of the paracatadioptric system relative to its mounting frame were selected as follows

$R_{m}=I_{3} \quad x_{m}=\left[\begin{array}{lll}0 & 0 & 0.1\end{array}\right]^{T} \quad[\mathrm{~m}]$

where $I_{3} \in \mathbb{R}^{3 \times 3}$ denotes a standard identity matrix. $\theta_{\max }$, and $\theta_{\text {min }}$ given in (51) were utilized to find the vertices of the hypercube $\Theta$, which is a cube in this case with its 8 vertices at $\left[\begin{array}{lll}0 & 0 & 0\end{array}\right]^{T}$, $\left[\begin{array}{lll}0 & 4 & 0\end{array}\right]^{T},\left[\begin{array}{lll}0 & 4 & 4\end{array}\right]^{T},\left[\begin{array}{lll}0 & 0 & 4\end{array}\right]^{T},\left[\begin{array}{ccc}4 & 0 & 0\end{array}\right]^{T},\left[\begin{array}{lll}4 & 0 & 4\end{array}\right]^{T}$, $\left[\begin{array}{lll}4 & 4 & 4\end{array}\right]^{T}$, and $\left[\begin{array}{lll}4 & 4 & 0\end{array}\right]^{T}$. Hence, a tetrahedron simplex $\Theta_{s}$, enclosing $\Theta$ was constructed, as shown in Fig. 2, whose vertices were given as follows

$\theta_{s 1}=\left[\begin{array}{lll}0 & 0 & 0\end{array}\right]^{T} \quad \theta_{s 3}=\left[\begin{array}{lll}16 & 0 & 0\end{array}\right]^{T}$

$\theta_{s 2}=\left[\begin{array}{lll}0 & 0 & 8\end{array}\right]^{T} \quad \theta_{s 4}=\left[\begin{array}{lll}0 & 16 & 0\end{array}\right]^{T}$.

Definition 1 was utilized to determine the concavity/convexity of the function $q(\cdot)$ on $\Theta_{s}$. Initializing the estimator as $\hat{\theta}\left(t_{0}\right)=$ $\left[\begin{array}{lll}0.5 & 1 & 1\end{array}\right]^{T}$ (i.e., $50 \%$ of the true values), and setting $f=0.5$ along with the simulation parameters given in (49), (50) and (52), gave the following initial values

$z\left(t_{0}\right)=1.9 \quad[\mathrm{~m}] \quad \hat{z}\left(t_{0}\right)=0.9 \quad[\mathrm{~m}]$.

The parameter $\alpha$ was set as $\alpha=5$, and $\varepsilon$ was selected as $\varepsilon=0.001$ to make the tuning error $\tilde{q}_{f \varepsilon}(t)$ introduced in (29) very close to $\tilde{q}_{f}(t)$ so that a high precision for the estimation is obtained.

Two different cases were considered in the simulation study, without changing any of the above mentioned parameters: case 1 was without any noise and case 2 was with additive-whiteGaussian-noise (AWGN) injected into the measured coordinates $u(t)$ and $v(t)$ using the awgn() function of Matlab. A constant signal-to-noise ratio (SNR) of 20 was maintained. It should be noted that injection of noise into $u(t)$ and $v(t)$ induces noise into $q(\theta, \Pi)$; hence, noise in any measurable signal contained in $\Pi(\cdot)$ is considered.

Fig. 3 shows $\hat{\theta}(t)$, the estimates of the 3D Euclidean coordinates of the object feature relative to the world frame, for case 1 . The range estimation error (i.e, $z(t)-\hat{z}(t))$ for case 1 is shown in Fig. 4. It can be seen from these figures that the developed estimation technique provides an accurate estimation of the range, with an accurate estimate of the 3D coordinates of the object feature relative to the world frame. Figs. 5 and 6 show $\hat{\theta}(t)$ and the range estimation error, respectively in the presence of noise (i.e., case 2). It can be inferred from these figures that the proposed range estimation technique is robust to noisy measurements and provides good estimates for the constant 3D coordinates of the object feature relative to the world frame, along with an estimate of its range. It is worthwhile to note that $\hat{\theta}(t)$ can be used to obtain other 3D coordinates of the object feature relative to $\mathcal{M}, \hat{x}(t), \hat{y}(t)$ from (19) and (20), respectively. a

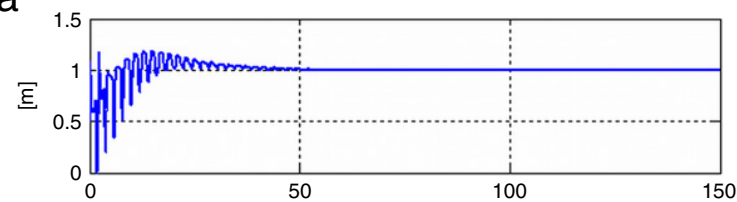

b

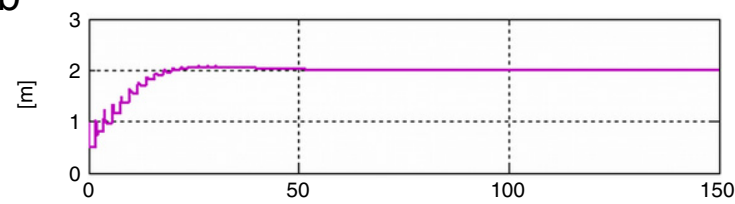

C

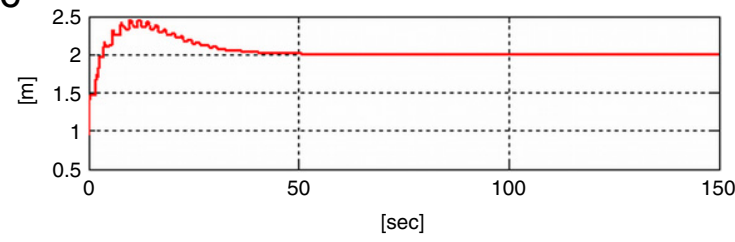

Fig. 3. Simulation case 1 : (a) $\hat{\theta}_{1}(t)$, (b) $\hat{\theta}_{2}(t)$, and (c) $\hat{\theta}_{3}(t)$.

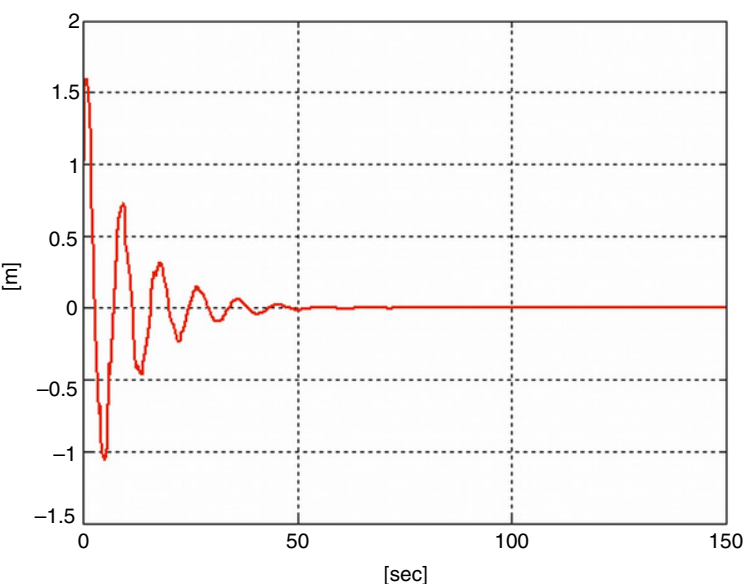

Fig. 4. Simulation case 1: range estimation error $z(t)-\hat{z}(t)$.

a

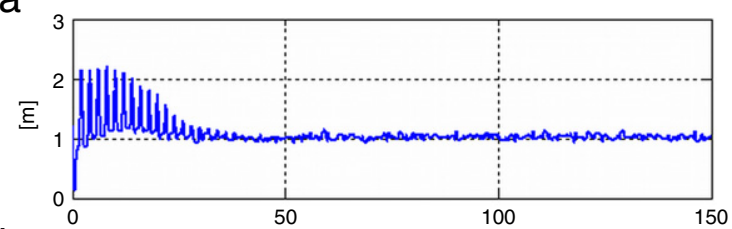

b

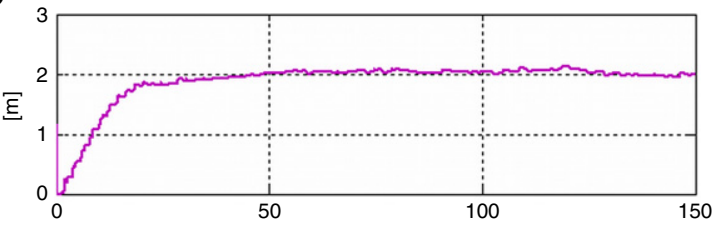

C

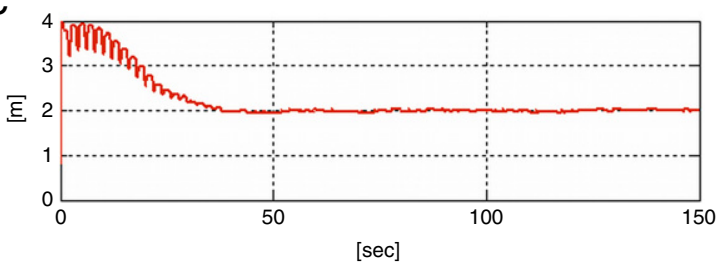

Fig. 5. Simulation case $2:$ (a) $\hat{\theta}_{1}(t)$, (b) $\hat{\theta}_{2}(t)$, and (c) $\hat{\theta}_{3}(t)$. 


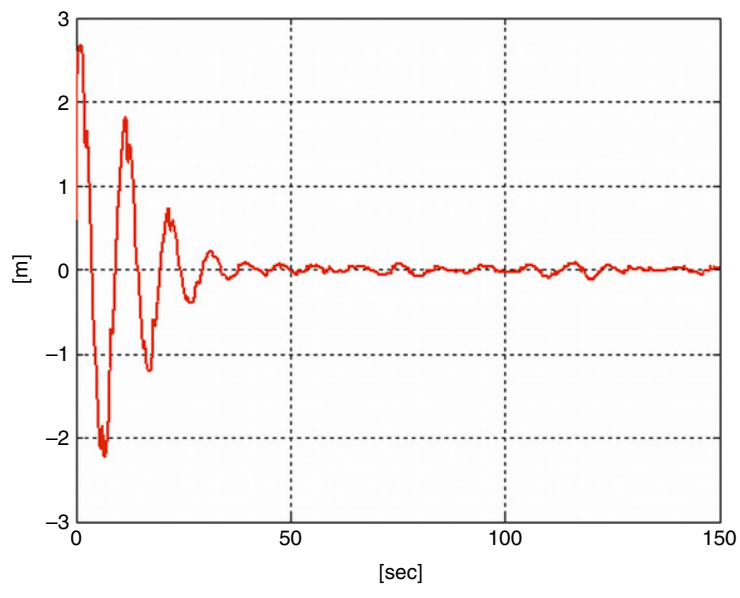

Fig. 6. Simulation case 2 : range estimation error $z(t)-\hat{z}(t)$.

Remark 13. The NLPE condition, given in (47), guarantees the parameter but it is not clear what role the NLPE condition plays in the rate of convergence. Furthermore, we note that the simulation results indicate that the constant parameter $\alpha$, defined in (24), can also be tuned to affect the rate of convergence at the cost of estimation accuracy. In this paper, we primarily focus on the architecture and the technique to identify the range for a paracatadioptric system. This technique can be further applied to any moving mechanical platform as long as its position and velocity signals are continuous, bounded, and the required assumptions are satisfied.

Remark 14. The initial range and the initial range estimate depend upon the actual values of the parameters and the initial estimate of the unknown parameter vector, respectively. If the initial error is high, the range estimation error will converge more slowly.

\section{Conclusion}

A novel technique for range identification and recovering the 3D Euclidean coordinates of a static object feature with a calibrated paracatadioptric system mounted on a moving platform with measurable position was presented. An adaptive estimator for a nonlinearly parameterized function of projected pixel coordinates was presented which facilitated the range estimation along with the estimation of 3D Euclidean coordinates of an object feature. A Lyapunov-type stability analysis was presented to prove that the proposed estimator is stable, and ensures global boundedness of the error signals. Further, the parameter estimation error signals were shown to be bounded by a desired precision upon satisfaction of an NLPE condition. Numerical simulation results were presented to demonstrate the efficiency of the developed range identification technique and its robustness to noise. The results show that the developed estimator can be used for range identification for applications with paracatadioptric systems where position measurements are readily available.

\section{Appendix A. Proof of Theorem 1}

Proof. To facilitate the proof, ${ }^{3}$ a nonnegative Lyapunov function $V(t) \in \mathbb{R}$ is defined as follows

$V=\frac{1}{2} \tilde{q}_{f \varepsilon}^{2}+\frac{1}{2} \tilde{\theta}^{T} \tilde{\theta}$

\footnotetext{
3 The proof follows the concept outlined in Annaswamy et al. (1998). We include it in a detailed manner for the sake of completeness.
}

The time derivative of (A.1) can be obtained as follows

$\dot{V}=\tilde{q}_{f \varepsilon} \dot{\tilde{q}}_{f \varepsilon}+\tilde{\theta}^{T} \dot{\tilde{\theta}}$

After utilizing the time derivative of (36), the expression given in (A.2) can be written as follows

$\dot{V}=\tilde{q}_{f \varepsilon} \dot{\tilde{q}}_{f \varepsilon}+\tilde{\theta}^{T} \dot{\hat{\theta}}$.

After substituting (31) into (A.3), the following expression is obtained

$\dot{V}=\tilde{q}_{f \varepsilon} \dot{\tilde{q}}_{f \varepsilon}+\tilde{\theta}^{T} \operatorname{Proj}\left\{-\tilde{q}_{f \varepsilon} \phi^{*}\right\}$.

It should be noted that an adaptive law with the projection algorithm defined on a convex set retains all the properties of the adaptive law without the projection algorithm (Ioannou \& Sun, 1996). The projection strategy given in (32) is on the cube $\Theta$ (i.e., a convex set); hence, the expression given in (A.4) can be written as follows

$\dot{V}=\tilde{q}_{f \varepsilon} \dot{\tilde{q}}_{f \varepsilon}-\tilde{\theta}^{T} \tilde{q}_{f \varepsilon} \phi^{*}$.

The expression given in (A.5) is rearranged as follows

$\dot{V}=\tilde{q}_{f \varepsilon}\left[\dot{\tilde{q}}_{f \varepsilon}-\tilde{\theta}^{T} \phi^{*}\right]$.

Two different cases are considered, Case I when $\left|\tilde{q}_{f}\right| \leq \varepsilon$, and Case II when $\forall\left|\tilde{q}_{f}\right|>\varepsilon$.

Case (I) From Remark 7 it follows that

$\dot{V}=0 \quad \forall\left|\tilde{q}_{f}\right| \leq \varepsilon$.

Case (II) Also, from Remark 7 and (A.6), the following expression can be obtained

$\dot{V}=\tilde{q}_{f \varepsilon}\left[\dot{\tilde{q}}_{f}-\tilde{\theta}^{T} \phi^{*}\right] \quad \forall\left|\tilde{q}_{f}\right|>\varepsilon$.

After substituting (30) into (A.8), the following expression is obtained

$\dot{V}=\tilde{q}_{f \varepsilon}\left[-\alpha \tilde{q}_{f \varepsilon}+\hat{q}-q-a^{*} \operatorname{sat}(r)-\tilde{\theta}^{T} \phi^{*}\right]$.

The inequality given in (A.9) can be rearranged as follows

$\dot{V}=-\alpha \tilde{q}_{f \varepsilon}^{2}+\tilde{q}_{f \varepsilon}\left[\hat{q}-q-\tilde{\theta}^{T} \phi^{*}-a^{*} \operatorname{sat}(r)\right]$.

Now two distinct sub-cases of Case II are considered: (a) when $\tilde{q}_{f}>\varepsilon$, and (b) when $\tilde{q}_{f}<-\varepsilon$.

(a) When $\tilde{q}_{f}>\varepsilon$, from (28) and (29) it follows that $\tilde{q}_{f \varepsilon}>0$ and $\operatorname{sat}(r)=\operatorname{sgn}\left(\tilde{q}_{f}\right)=1$. After utilizing (A.10), $\dot{V}(t)$ can be written as follows

$\dot{V}=-\alpha \tilde{q}_{f \varepsilon}^{2}+\tilde{q}_{f \varepsilon}\left[\hat{q}-q-\tilde{\theta}^{T} \phi^{*}-a^{*}\right]$.

It follows from (A.11) that $\dot{V}(t) \leq 0$ if the following inequality holds

$a^{*} \geq \hat{q}-q-\tilde{\theta}^{T} \phi^{*} \quad \forall \theta \in \Theta_{s}$.

Therefore, we choose to maximize $a^{*}(t)$ as follows

$a^{*}=\max _{\theta \in \Theta_{S}}\left[\hat{q}-q-\tilde{\theta}^{T} \phi^{*}\right]$ for any $\phi^{*}$.

Since, $a^{*}(t)$ is like a gain in (30), we seek to find $\phi^{*}(t)$ so that $a^{*}(t)$ is minimized; thus, $a^{*}(t)$ is chosen as follows

$a^{*}=\min _{\phi \in \mathbb{R}^{3}} \max _{\theta \in \Theta_{s}}\left[\hat{q}-q-\tilde{\theta}^{T} \phi^{*}\right]$. 
(b) When $\tilde{q}_{f}<-\varepsilon$, from (28) and (29) it follows that $\tilde{q}_{f \varepsilon}<0$ and $\operatorname{sat}(r)=\operatorname{sgn}\left(\tilde{q}_{f}\right)=-1$. After utilizing (A.10), $\dot{V}(t)$ can be written as follows

$\dot{V}=-\alpha \tilde{q}_{f \varepsilon}^{2}+\tilde{q}_{f \varepsilon}\left[\hat{q}-q-\tilde{\theta}^{T} \phi^{*}+a^{*}\right]$.

From (A.15) it follows that $\dot{V}(t) \leq 0$ if the following inequality holds

$a^{*} \geq q-\hat{q}+\tilde{\theta}^{T} \phi^{*} \quad \forall \theta \in \Theta_{s}$.

Following along the same lines as in (a), the following expression can be written

$a^{*}=\min _{\phi \in \mathbb{R}^{3}} \max _{\theta \in \Theta_{s}}\left[q-\hat{q}+\tilde{\theta}^{T} \phi^{*}\right]$.

After combining (A.14) and (A.17), the following expression is obtained

$a^{*}=\min _{\phi \in \mathbb{R}^{3}} \max _{\theta \in \Theta_{S}} \operatorname{sat}(r)\left[\hat{q}-q-\tilde{\theta}^{T} \phi^{*}\right]$.

After utilizing (A.12) and (A.16) the following inequality can be obtained

$\operatorname{sat}(r)\left[\hat{q}-q-\tilde{\theta}^{T} \phi^{*}\right]-a^{*} \leq 0$.

The expression given in (A.11) can be rewritten as follows

$\dot{V}=-\alpha \tilde{q}_{f \varepsilon}^{2}+\tilde{q}_{f \varepsilon} \operatorname{sat}(r)\left\{\operatorname{sat}(r)\left[\hat{q}-q-\tilde{\theta}^{T} \phi^{*}\right]-a^{*}\right\}$.

Thus, after utilizing (A.19), and the fact that $\tilde{q}_{f \varepsilon} \operatorname{sat}(r) \geq 0$ when $\left|\tilde{q}_{f}\right|>\varepsilon, \dot{V}(t)$ can be upper bounded as follows

$\dot{V} \leq-\alpha \tilde{q}_{f \varepsilon}^{2} \quad \forall\left|\tilde{q}_{f}\right|>\varepsilon$.

After integrating (A.21), the following inequality can be obtained

$\alpha \int_{t_{0}}^{\infty} \tilde{q}_{f \varepsilon}^{2} \mathrm{~d} \tau<V\left(t_{0}\right)-V(\infty)$

From (A.1), (A.7) and (A.21), it can be concluded that $V(t) \in \mathcal{L}_{\infty}$. From (A.22), it is clear that $\tilde{q}_{f \varepsilon}(t) \in \mathscr{L}_{2} \cap \mathcal{L}_{\infty}$; thus, from (29), it can be concluded that $\tilde{q}_{f}(t) \in \mathcal{L}_{\infty}$. Since the projection strategy given in (32) ensures $\hat{\theta}(t) \in \Theta_{s}$; thus, it follows that $\hat{\theta}(t) \in \mathcal{L}_{\infty}$. Hence, from (22), it follows that $\hat{q}(\cdot) \in \mathcal{L}_{\infty}$. Since $a^{*}(t)$ is a function of the bounded signals, and $q(\cdot)$ is a measurable bounded signal, from (30), it follows that $\dot{\tilde{q}}_{f}(t) \in \mathcal{L}_{\infty}$. It is clear from the projection strategy that $\dot{\hat{\theta}}(t) \in \mathcal{L}_{\infty}$; thus, from (36), $\dot{\tilde{\theta}}(t) \in \mathcal{L}_{\infty}$.

\section{Appendix B. Proof of Theorem 2}

Proof. To facilitate the proof, ${ }^{4}$ without loss of generality, we assume $\beta\left(\Pi\left(t_{2}\right)\right)=1$ i.e., $q\left(\theta, \Pi\left(t_{2}\right)\right)$ is convex on $\Theta_{s}{ }^{5}$ Thus, the expression given in (47) can be rewritten as follows

$q\left(\hat{\theta}\left(t_{1}\right), \Pi\left(t_{2}\right)\right)-q\left(\theta, \Pi\left(t_{2}\right)\right) \geq \bar{\varepsilon}$

where $\bar{\varepsilon}=\varepsilon_{u}\left\|\hat{\theta}\left(t_{1}\right)-\theta\right\|$. To further facilitate the proof, we define a region of convergence as follows

$\Omega_{\varepsilon}=\{d: V(d) \leq \gamma\}$

\footnotetext{
4 The proof follows the concept outlined in Cao et al. (2003). We include it in a detailed manner for the sake of completeness.

5 A similar proof can be given if $\beta\left(\Pi\left(t_{2}\right)\right)=-1$, i.e., $q(\cdot)$ is concave on $\Theta_{s}$.
}

where

$d=\left[\begin{array}{ll}\tilde{q}_{f \varepsilon} & \tilde{\theta}^{T}\end{array}\right]^{T}$

and $V(\cdot)$ is the Lyapunov function defined in (A.1).

From the region of convergence, we know that if $d\left(t_{1}\right) \in \Omega_{\varepsilon}$ then $d(t)$ for all $t \geq t_{1}$ stays in $\Omega_{\varepsilon}$. Also, $V(\cdot)$ is a Lyapunov function and its time derivative is always non-positive (see Appendix A); hence, we assume that $d\left(t_{1}\right) \notin \Omega_{\varepsilon}$. The proof of this theorem follows by showing that $V(\cdot)$ decreases by a finite amount over every interval of time until the trajectories reach $\Omega_{\varepsilon}$.

If $d\left(t_{1}\right) \notin \Omega_{\varepsilon}$, from (B.2), it is clear that $V(\cdot)>\gamma$. Hence, after utilizing (A.1), (B.2) and (B.3), $V(\cdot)$ can be expressed as follows

$V=\frac{1}{2} \tilde{q}_{f \varepsilon}^{2}+\frac{1}{2} \tilde{\theta}^{T} \tilde{\theta}>\gamma$.

From (B.4), it is clear that the following inequalities are not satisfied simultaneously

$\left|\tilde{q}_{f \varepsilon}\left(t_{1}\right)\right|<\sqrt{\gamma}$

$\left\|\tilde{\theta}\left(t_{1}\right)\right\|<\sqrt{\gamma}$.

It can be seen that if the inequalities given in (B.5) and (B.6) are satisfied simultaneously, then $V(\cdot) \leq \gamma$, which is not true; thus, we have three possible cases as follows

1. $\left|\tilde{q}_{f \varepsilon}\left(t_{1}\right)\right|>\sqrt{\gamma}$ or

2. $\left\|\tilde{\theta}\left(t_{1}\right)\right\|>\sqrt{\gamma}$ or

3. $\left|\tilde{q}_{f \varepsilon}\left(t_{1}\right)\right|>\sqrt{\gamma}$ and $\left\|\tilde{\theta}\left(t_{1}\right)\right\|>\sqrt{\gamma}$.

If case 1 or case 3 holds, since $\left|\tilde{q}_{f \varepsilon}\left(t_{1}\right)\right|>\sqrt{\gamma}$, from Property 1 (see Nath, Tatlicioglu, \& Dawson, 2009b), it is clear that $V(\cdot)$ decreases. If case 2 holds, then we show in the following analysis that $\left|\tilde{q}_{f \varepsilon}(t)\right|$ becomes large for some $t>t_{1}$ and $V(\cdot)$ decreases.

After taking the square of the right-hand side of (B.1), the following inequality can be obtained

$\bar{\varepsilon}^{2} \geq \varepsilon_{u}^{2} \gamma$

Substituting (48) into (B.7) results in the following inequality

$\bar{\varepsilon}^{2} \geq 8 \varepsilon c_{1}$.

We show that if (B.8) holds, then there exists a time $t_{3} \in\left[t_{2}, t_{2}+T_{1}\right]$ such that

$\left|\tilde{q}_{f \varepsilon}\left(t_{3}\right)\right|>\min \{1, \bar{\delta}\}$

where

$\bar{\delta}=\min \left\{\frac{\bar{\varepsilon}}{2 c_{2}}, \frac{\bar{\varepsilon}^{2}-4 \varepsilon c_{1}}{2 \bar{\varepsilon} c_{2}+4 c_{1}}\right\}$

where

$c_{2}=L_{2} B_{\phi} T_{0}+\alpha ; \quad T_{1}=\frac{\bar{\varepsilon}-\bar{\delta} c_{2}}{c_{1}}$.

We prove by contradiction that (B.9) holds. To facilitate the proof, we consider the following inequality

$\left|\tilde{q}_{f \varepsilon}\left(t_{2}+\tau\right)\right|<\min \{1, \bar{\delta}\} \quad \forall \tau \in\left[0, T_{1}\right]$.

The expression given in (30) can be rewritten as follows

$\dot{\tilde{q}}_{f}\left(t_{2}+\tau\right) \geq-\alpha \min \{1, \bar{\delta}\}+q\left(\hat{\theta}, \Pi\left(t_{2}+\tau\right)\right)$

$-q\left(\theta, \Pi\left(t_{2}+\tau\right)\right)-a^{*} \operatorname{sat}(r)$ 
where (B.12) was utilized. We seek to establish lower bounds for $\left[q\left(\hat{\theta}, \Pi\left(t_{2}+\tau\right)\right)-q\left(\theta, \Pi\left(t_{2}+\tau\right)\right)\right]$ and $-a^{*} \operatorname{sat}(r)$ in (B.13) in order to prove that $\tilde{q}_{f \varepsilon}(t)$ becomes large over $\left[t_{2}, t_{2}+T_{1}\right]$. From Assumption 5, it follows that

$$
\left|q\left(\theta+\Delta \theta, \Pi\left(t_{2}\right)\right)-q\left(\theta, \Pi\left(t_{2}\right)\right)\right| \leq L_{2}\|\Delta \theta\| .
$$

After integrating (31) from $t_{1}$ to $t_{2}$, the following expression is obtained

$\hat{\theta}\left(t_{2}\right)-\hat{\theta}\left(t_{1}\right)=\int_{t_{1}}^{t_{2}}-\tilde{q}_{f \varepsilon}(\sigma) \phi^{*}(\sigma) \mathrm{d} \sigma$.

After taking the norm on both the sides of (B.15), the following inequality can be obtained

$$
\left\|\hat{\theta}\left(t_{2}\right)-\hat{\theta}\left(t_{1}\right)\right\| \leq \int_{t_{1}}^{t_{2}}\left\|\tilde{q}_{f \varepsilon}(\sigma)\right\|\left\|\phi^{*}(\sigma)\right\| \mathrm{d} \sigma .
$$

The left-hand side of (B.16) can be upper bounded as follows

$$
\left\|\hat{\theta}\left(t_{2}\right)-\hat{\theta}\left(t_{1}\right)\right\| \leq \min \{1, \bar{\delta}\} L_{\phi} T_{0}
$$

where (B.12), Remark 9, and the fact that $T_{0} \geq t_{2}-t_{1}$ were utilized. After utilizing (B.14) and (B.17), the following inequality can be obtained

$$
\left|q\left(\hat{\theta}\left(t_{2}\right), \Pi\left(t_{2}\right)\right)-q\left(\hat{\theta}\left(t_{1}\right), \Pi\left(t_{2}\right)\right)\right| \leq L_{2} \min \{1, \bar{\delta}\} L_{\phi} T_{0} .
$$

From (B.18), it follows that

$$
-L_{2} \min \{1, \bar{\delta}\} L_{\phi} T_{0} \leq q\left(\hat{\theta}\left(t_{2}\right), \Pi\left(t_{2}\right)\right)-q\left(\hat{\theta}\left(t_{1}\right), \Pi\left(t_{2}\right)\right) .
$$

After adding (B.1) and (B.19), the following expression is obtained $\bar{\varepsilon}-L_{2} \min \{1, \bar{\delta}\} L_{\phi} T_{0} \leq q\left(\hat{\theta}\left(t_{2}\right), \Pi\left(t_{2}\right)\right)-q\left(\theta, \Pi\left(t_{2}\right)\right)$.

From Assumption 4, it follows that

$$
\left\|\Pi\left(t_{2}+\tau\right)-\Pi\left(t_{2}\right)\right\| \leq L_{1} \tau \quad \forall \tau \in\left[0, T_{1}\right] .
$$

Thus, the following inequalities can be obtained

$$
\begin{aligned}
& \left|q\left(\theta, \Pi\left(t_{2}+\tau\right)\right)-q\left(\theta, \Pi\left(t_{2}\right)\right)\right| \\
& \quad \leq L_{2}\left(\left\|\Pi\left(t_{2}+\tau\right)-\Pi\left(t_{2}\right)\right\|\right) \\
& \quad \leq L_{2} L_{1} \tau .
\end{aligned}
$$

From (B.22) and (B.23), the following expression is obtained

$q\left(\theta, \Pi\left(t_{2}+\tau\right)\right)-q\left(\theta, \Pi\left(t_{2}\right)\right) \leq L_{2} L_{1} \tau$

which can be rewritten as follows

$q\left(\theta, \Pi\left(t_{2}\right)\right)-q\left(\theta, \Pi\left(t_{2}+\tau\right)\right) \geq-L_{2} L_{1} \tau$.

After combining (B.17), (B.21), and Assumption 5, the following expression can be obtained

$$
\begin{aligned}
& \left|q\left(\hat{\theta}\left(t_{2}+\tau\right), \Pi\left(t_{2}+\tau\right)\right)-q\left(\hat{\theta}\left(t_{2}\right), \Pi\left(t_{2}\right)\right)\right| \\
& \quad \leq L_{2} L_{1} \tau+L_{2} L_{\phi} \tau
\end{aligned}
$$

where the fact that $\min (a, b) \leq a$ and $\min (a, b) \leq b$ was utilized. From (B.26), it follows that

$$
\begin{gathered}
-L_{2} L_{1} \tau-L_{2} L_{\phi} \tau \leq \\
q\left(\hat{\theta}\left(t_{2}+\tau\right), \Pi\left(t_{2}+\tau\right)\right) \\
-q\left(\hat{\theta}\left(t_{2}\right), \Pi\left(t_{2}\right)\right) .
\end{gathered}
$$

After adding (B.25) and (B.27), the following expression is obtained

$$
\begin{aligned}
-L_{2}\left(2 L_{1}+L_{\phi}\right) \tau \leq & q\left(\hat{\theta}\left(t_{2}+\tau\right), \Pi\left(t_{2}+\tau\right)\right) \\
& -q\left(\theta, \Pi\left(t_{2}+\tau\right)\right)+q\left(\theta, \Pi\left(t_{2}\right)\right) \\
& -q\left(\hat{\theta}\left(t_{2}\right), \Pi\left(t_{2}\right)\right) .
\end{aligned}
$$

After rearranging (B.28), the following expression can be obtained

$$
\begin{gathered}
-L_{2}\left(2 L_{1}+L_{\phi}\right) \tau+q\left(\hat{\theta}\left(t_{2}\right), \Pi\left(t_{2}\right)\right)-q\left(\theta, \Pi\left(t_{2}\right)\right) \\
\leq q\left(\hat{\theta}\left(t_{2}+\tau\right), \Pi\left(t_{2}+\tau\right)\right)-q\left(\theta, \Pi\left(t_{2}+\tau\right)\right) .
\end{gathered}
$$

Utilizing (B.20) and (B.29) results in the following expression

$$
\begin{aligned}
\bar{\varepsilon} & -L_{2} \min \{1, \bar{\delta}\} L_{\phi} T_{0}-L_{2}\left(2 L_{1}+L_{\phi}\right) \tau \\
& \leq q\left(\hat{\theta}\left(t_{2}+\tau\right), \Pi\left(t_{2}+\tau\right)\right)-q\left(\theta, \Pi\left(t_{2}+\tau\right)\right) .
\end{aligned}
$$

Thus, a lower bound on the term $\left[q\left(\hat{\theta}, \Pi\left(t_{2}+\tau\right)\right)-q\left(\theta, \Pi\left(t_{2}+\tau\right)\right)\right]$ in (B.13) is established. Now, we seek to find a lower bound on the term $-a^{*} \operatorname{sat}(r)$ in (B.13). After changing the variable $t_{2}$ to $t_{2}+\tau$ and $t_{1}$ to $t_{2}$, the expression given in (B.17) can be rewritten as follows

$$
\left\|\hat{\theta}\left(t_{2}+\tau\right)-\hat{\theta}\left(t_{2}\right)\right\| \leq \min \{1, \bar{\delta}\} L_{\phi} \tau .
$$

After multiplying (B.31) by $\phi^{*}\left(t_{2}\right)$ and utilizing Remark 9, the following expression is obtained

$$
\left|\phi^{*}\left(t_{2}\right)\left(\hat{\theta}\left(t_{2}+\tau\right)-\hat{\theta}\left(t_{2}\right)\right)\right| \leq \min \{1, \bar{\delta}\} L_{\phi}^{2} \tau \text {. }
$$

From Property 3 (see Nath et al., 2009b), it follows that

$a_{+}^{*}\left(\hat{\theta}\left(t_{2}\right), \Pi\left(t_{2}\right)\right)=0$

when $\beta\left(\Pi\left(t_{2}\right)\right)=1$ where $a_{+}^{*}(\cdot)$ denotes $a^{*}(t)$ when $\tilde{q}_{f \varepsilon}>0$ (see Nath et al., 2009b). From (33), the following expression is obtained

$a_{+}^{*}\left(\hat{\theta}\left(t_{2}\right), \Pi\left(t_{2}\right)\right)=\max \left\{\hat{q}_{2}-\phi^{*}\left(t_{2}\right)\left(\hat{\theta}\left(t_{2}\right)-\theta\right)\right\}$

where

$\hat{q}_{2}=q\left(\hat{\theta}\left(t_{2}\right), \Pi\left(t_{2}\right)\right)-q\left(\theta, \Pi\left(t_{2}\right)\right)$.

At time instant $t_{2}+\tau$, the expression given in (B.34) can be written as follows

$$
\begin{aligned}
& a_{+}^{*}\left(\hat{\theta}\left(t_{2}+\tau\right), \Pi\left(t_{2}+\tau\right)\right) \\
& \quad=\max \left\{\hat{q}_{2 \tau}-\phi^{*}\left(t_{2}+\tau\right)\left(\hat{\theta}\left(t_{2}+\tau\right)-\theta\right)\right\}
\end{aligned}
$$

where

$\hat{q}_{2 \tau}=q\left(\hat{\theta}\left(t_{2}+\tau\right), \Pi\left(t_{2}+\tau\right)\right)-q\left(\theta, \Pi\left(t_{2}+\tau\right)\right)$.

Since $\phi^{*}\left(t_{2}+\tau\right)$ results in the minimum value of $a_{+}^{*}\left(\hat{\theta}\left(t_{2}+\right.\right.$ $\left.\tau), \Pi\left(t_{2}+\tau\right)\right)$, the left-hand side of (B.36) can be upper bounded as follows

$a_{+}^{*}\left(\hat{\theta}\left(t_{2}+\tau\right), \Pi\left(t_{2}+\tau\right)\right)$

$$
\leq \max \left\{\hat{q}_{2 \tau}-\phi^{*}\left(t_{2}\right)\left(\hat{\theta}\left(t_{2}+\tau\right)-\theta\right)\right\} .
$$

After adding and subtracting the terms $\hat{q}_{2}$ and $\phi^{*}\left(t_{2}\right) \hat{\theta}\left(t_{2}\right)$ to the right-hand side of (B.38), and then simplifying results in the following expression

$$
\begin{aligned}
& a_{+}^{*}\left(\hat{\theta}\left(t_{2}+\tau\right), \Pi\left(t_{2}+\tau\right)\right) \\
& \quad \leq \max \left\{\hat{q}_{2 \tau}-\hat{q}_{2}-\phi^{*}\left(t_{2}\right)\left(\hat{\theta}\left(t_{2}+\tau\right)-\hat{\theta}\left(t_{2}\right)\right)\right\} \\
& \quad+\max \left\{\hat{q}_{2}-\phi^{*}\left(t_{2}\right)\left(\hat{\theta}\left(t_{2}\right)-\theta\right)\right\}
\end{aligned}
$$


where the fact that $\max (a+b) \leq \max (a)+\max (b)$ was utilized. After utilizing (B.34), the expression given in (B.39) can be written as follows

$$
\begin{aligned}
& a_{+}^{*}\left(\hat{\theta}\left(t_{2}+\tau\right), \Pi\left(t_{2}+\tau\right)\right) \leq \max \left\{\hat{q}_{2 \tau}-\hat{q}_{2}\right. \\
& \left.\quad-\phi^{*}\left(t_{2}\right)\left(\hat{\theta}\left(t_{2}+\tau\right)-\hat{\theta}\left(t_{2}\right)\right)\right\}+a_{+}^{*}\left(\hat{\theta}\left(t_{2}\right), \Pi\left(t_{2}\right)\right) .
\end{aligned}
$$

The expression given in (B.40) can be upper bounded as follows

$$
\begin{aligned}
& a_{+}^{*}\left(\hat{\theta}\left(t_{2}+\tau\right), \Pi\left(t_{2}+\tau\right)\right) \leq \max \left\{\hat{q}_{2 \tau}-\hat{q}_{2}\right\} \\
& \quad+\max \left\{-\phi^{*}\left(t_{2}\right)\left(\hat{\theta}\left(t_{2}+\tau\right)-\hat{\theta}\left(t_{2}\right)\right)\right\} \\
& \quad+a_{+}^{*}\left(\hat{\theta}\left(t_{2}\right), \Pi\left(t_{2}\right)\right) .
\end{aligned}
$$

The expression given in (B.41) can be rewritten as follows

$a_{+}^{*}\left(\hat{\theta}\left(t_{2}+\tau\right), \Pi\left(t_{2}+\tau\right)\right) \leq L_{2}\left(2 L_{1}+L_{\phi}\right) \tau+\min \{1, \bar{\delta}\} L_{\phi}^{2} \tau$

where (B.28), (B.32), (B.33), (B.35) and (B.37) were utilized. Since $\min \{1, \bar{\delta}\} \leq 1$, (B.42) can be rewritten as follows

$$
\begin{aligned}
a_{+}^{*}\left(\hat{\theta}\left(t_{2}+\tau\right), \Pi\left(t_{2}+\tau\right)\right) & \leq L_{2}\left(2 L_{1}+L_{\phi}\right) \tau+L_{\phi}^{2} \tau \\
& \leq\left(2 L_{2} L_{1}+L_{2} L_{\phi}+L_{\phi}^{2}\right) \tau .
\end{aligned}
$$

The inequality given in (B.43) is rewritten as follows

$a_{+}^{*}\left(\hat{\theta}\left(t_{2}+\tau\right), \Pi\left(t_{2}+\tau\right)\right) \operatorname{sat}(r) \leq\left(2 L_{2} L_{1}+L_{2} L_{\phi}+L_{\phi}^{2}\right) \tau$

where the fact that sat $(r) \leq 1$ was utilized. After multiplying both the sides of (B.44) by -1 , and utilizing Property 2 (see Nath et al., $2009 \mathrm{~b})$, the lower bound on the term $-a^{*} \operatorname{sat}(r)$ in (30) is obtained as follows

$$
\begin{gathered}
-a^{*}\left(\hat{\theta}\left(t_{2}+\tau\right), \Pi\left(t_{2}+\tau\right)\right) \operatorname{sat}(r) \\
\geq-\left(2 L_{2} L_{1}+L_{2} L_{\phi}+L_{\phi}^{2}\right) \tau .
\end{gathered}
$$

Now, the expression given in (B.13) can be rewritten as follows

$$
\begin{aligned}
& \dot{\tilde{q}}_{f}\left(t_{2}+\tau\right) \geq-\alpha \min \{1, \bar{\delta}\}+\bar{\varepsilon}-L_{2} \min \{1, \bar{\delta}\} L_{\phi} T_{0} \\
& -L_{2}\left(2 L_{1}+L_{\phi}\right) \tau-\left(2 L_{2} L_{1}+L_{2} L_{\phi}+L_{\phi}^{2}\right) \tau
\end{aligned}
$$

where (B.30) and (B.45) were utilized. After substituting (48) and (B.11) into (B.46), the following expression can be obtained

$\dot{\tilde{q}}_{f}\left(t_{2}+\tau\right) \geq \bar{\varepsilon}-c_{2} \min \{1, \bar{\delta}\}-c_{1} \tau$.

Since $\min \{1, \bar{\delta}\} \leq \bar{\delta}, \dot{\tilde{q}}_{f}(t)$ can be lower bounded as follows

$\dot{\tilde{q}}_{f}\left(t_{2}+\tau\right) \geq c_{3}-c_{1} \tau$

where

$c_{3}=\bar{\varepsilon}-c_{2} \bar{\delta}$.

Integrating both the sides of (B.48) over [0, $\left.T_{1}\right]$, where $T_{1}$ is defined in (B.11) results in the following expression

$\int_{0}^{T_{1}} \dot{\tilde{q}}_{f}\left(t_{2}+\tau\right) \mathrm{d} \tau \geq\left.\left(c_{3} \tau-\frac{1}{2} c_{1} \tau^{2}\right)\right|_{0} ^{T_{1}}$.

Simplifying the right-hand side of (B.50) results in the following expression

$$
\left.\left(c_{3} \tau-\frac{1}{2} c_{1} \tau^{2}\right)\right|_{0} ^{T_{1}}=\frac{1}{2} \frac{c_{3}^{2}}{c_{1}}
$$

where (B.11) was utilized. After performing a change of variable $\rho=t_{2}+\tau$ on the left-hand side of (B.50), the following expressions can be obtained

$$
\begin{aligned}
\int_{0}^{T_{1}} \dot{q}_{f}\left(t_{2}+\tau\right) \mathrm{d} \tau & =\int_{t_{2}}^{t_{2}+T_{1}} \dot{\tilde{q}}_{f}(\rho) \mathrm{d} \rho \\
& =\left.\tilde{q}_{f}(\rho)\right|_{t_{2}} ^{t_{2}+T_{1}} \\
& =\tilde{q}_{f}\left(t_{2}+T_{1}\right)-\tilde{q}_{f}\left(t_{2}\right) .
\end{aligned}
$$

After combining (B.51) and (B.54), the expression given in (B.51) can be rewritten as follows

$\tilde{q}_{f}\left(t_{2}+T_{1}\right)-\tilde{q}_{f}\left(t_{2}\right) \geq \frac{1}{2} \frac{c_{3}^{2}}{c_{1}}$.

Taking $\tau=0$ in (B.12) results in the following expression

$$
-\min \{1, \bar{\delta}\}<\tilde{q}_{f \varepsilon}\left(t_{2}\right)<\min \{1, \bar{\delta}\} .
$$

The inequality given in (B.56) can be rewritten as follows

$$
-\varepsilon-\min \{1, \bar{\delta}\}<\tilde{q}_{f}\left(t_{2}\right)<\varepsilon+\min \{1, \bar{\delta}\}
$$

where (29) was utilized. After substituting (B.57) into (B.55), the following inequality can be written

$\tilde{q}_{f}\left(t_{2}+T_{1}\right) \geq \frac{c_{3}^{2}}{2 c_{1}}-\varepsilon-\min \{1, \bar{\delta}\}$.

Since $\min (a, b) \leq a$ and $\min (a, b) \leq b$, from the definition of $\bar{\delta}$ given in (B.10), the following inequality can be obtained

$\bar{\delta} \leq \frac{\bar{\varepsilon}^{2}-4 \varepsilon c_{1}}{2 \bar{\varepsilon} c_{2}+4 c_{1}}$.

After multiplying both the sides of (B.59) by the term $\left(2 \bar{\varepsilon} c_{2}+4 c_{1}\right)$, the following inequalities can be obtained

$2 \bar{\delta} \bar{\varepsilon} c_{2}+4 \bar{\delta} c_{1} \leq \bar{\varepsilon}^{2}-4 \varepsilon c_{1}$

$4 c_{1}(\bar{\delta}+\varepsilon) \leq \bar{\varepsilon}^{2}-2 \bar{\delta} \bar{\varepsilon} c_{2}$

$2(\bar{\delta}+\varepsilon) \leq \frac{\bar{\varepsilon}^{2}-2 \bar{\delta} \bar{\varepsilon} c_{2}}{2 c_{1}}$.

After adding and subtracting the term $\left(\bar{\delta} c_{2}\right)^{2}$ to the right-hand side of (B.60) results in the following expressions

$$
\begin{aligned}
\frac{\bar{\varepsilon}^{2}-2 \bar{\delta} \bar{\varepsilon} c_{2}}{2 c_{1}} & =\frac{\bar{\varepsilon}^{2}-2 \bar{\delta} \bar{\varepsilon} c_{2}+\left(\bar{\delta} c_{2}\right)^{2}-\left(\bar{\delta} c_{2}\right)^{2}}{2 c_{1}} \\
& =\frac{\left(\bar{\varepsilon}-\bar{\delta} c_{2}\right)^{2}-\left(\bar{\delta} c_{2}\right)^{2}}{2 c_{1}} \\
& =\frac{c_{3}^{2}-\left(\bar{\delta} c_{2}\right)^{2}}{2 c_{1}} .
\end{aligned}
$$

After utilizing (B.60) and (B.61), the following inequality can be obtained

$\frac{\left(\bar{\delta} c_{2}\right)^{2}}{2 c_{1}}+2(\bar{\delta}+\varepsilon) \leq \frac{c_{3}^{2}}{2 c_{1}}$.

After utilizing (B.62), the inequality given in (B.58) can be written as follows

$$
\begin{aligned}
\tilde{q}_{f}\left(t_{2}+T_{1}\right) & \geq \frac{\left(\bar{\delta} c_{2}\right)^{2}}{2 c_{1}}+2(\bar{\delta}+\varepsilon)-\varepsilon-\min \{1, \bar{\delta}\} \\
& \geq \frac{\left(\bar{\delta} c_{2}\right)^{2}}{2 c_{1}}+\bar{\delta}+\varepsilon+\bar{\delta}-\min \{1, \bar{\delta}\}
\end{aligned}
$$




$$
\begin{aligned}
& \geq \frac{\left(\bar{\delta} c_{2}\right)^{2}}{2 c_{1}}+\bar{\delta}+\varepsilon \\
& \geq \bar{\delta}+\varepsilon .
\end{aligned}
$$

From (29), it can be seen that the expression given in (B.63) implies that $\tilde{q}_{f \varepsilon} \geq \bar{\delta}$, which contradicts (B.12); thus, it can be easily concluded that (B.9) must hold.

Thus, it was shown that if $V\left(t_{1}\right)>\gamma$, then one of the following inequalities hold

$\left|\tilde{q}_{f \varepsilon}\left(t_{3}\right)\right| \geq \delta \min \{1, \bar{\delta}\} \quad \forall t_{3} \in\left[t_{1}, t_{1}+T_{0}+T_{1}\right]$

$\left|\tilde{q}_{f \varepsilon}\left(t_{1}\right)\right|>\sqrt{\gamma}$.

From Property 1 (see Nath et al., 2009b), it follows that if (B.64) holds, then

$V\left(t_{3}+T_{1}^{\prime}\right) \leq V\left(t_{3}\right)-\frac{\alpha \delta^{3}}{3(M+\alpha \delta)}$

where $T_{1}^{\prime}=\delta /(M+\alpha \delta)$. Similarly, if (B.65) holds, from Property 1 (see Nath et al., 2009b), it follows that

$V\left(t_{1}+T_{2}^{\prime}\right) \leq V\left(t_{1}\right)-\frac{\alpha \sqrt{\gamma}^{3}}{3(M+\alpha \sqrt{\gamma})}$

where $T_{2}^{\prime}=\sqrt{\gamma} /(M+\alpha \delta)$. Since $V(t)$ is a nonincreasing function, the following expression can be concluded from (B.66) and (B.67)

$V\left(t_{1}+T_{3}^{\prime}\right) \leq V\left(t_{1}\right)-\Delta V \quad \forall V\left(t_{1}\right)>\gamma$

where

$T_{3}^{\prime}=\max \left\{T_{0}+T_{1}+T_{1}^{\prime}, T_{0}+T_{1}+T_{2}^{\prime}\right\}$

$\Delta V=\min \left\{\frac{\alpha \delta^{3}}{3(M+\alpha \delta)}, \frac{\alpha \sqrt{\gamma}^{3}}{3(M+\alpha \sqrt{\gamma})}\right\}$.

Thus, it is clear from (B.68) that $V(t)$ decreases by a finite amount over every interval $T_{3}^{\prime}$ until trajectories reach $\Omega_{\varepsilon}$; hence, from (A.1), (B.2) and (B.3), it follows that $\|\tilde{\theta}(t)\| \leq \sqrt{\gamma}$ as $t \rightarrow \infty$.

\section{Appendix C. Validity of Assumptions 4 and 5}

Assumptions 4 and 5 are technical assumptions that are used for the proof of convergence as given in Cao et al. (2003). In general, it is not possible to ascertain whether these assumptions are realistic for the problem attacked in this paper; however, in this appendix we give an argument which gives confidence that the assumptions have some validity with regard to the estimation problem.

To facilitate the validity argument, we add and subtract $q\left(\theta_{0}+\right.$ $\left.\Delta \theta_{0}, \Pi\right)$ to the left-hand side of (16) to obtain the following expression

$$
\begin{aligned}
& \left|q\left(\theta_{0}+\Delta \theta_{0}, \Pi+\Delta \Pi\right)-q\left(\theta_{0}, \Pi\right)\right|=\mid q\left(\theta_{0}+\Delta \theta_{0}, \Pi+\Delta \Pi\right) \\
& -q\left(\theta_{0}+\Delta \theta_{0}, \Pi\right)+q\left(\theta_{0}+\Delta \theta_{0}, \Pi\right)-q\left(\theta_{0}, \Pi\right) \mid .
\end{aligned}
$$

The left-hand side of (C.1) can be upper bounded as follows

$$
\begin{aligned}
& \left|q\left(\theta_{0}+\Delta \theta_{0}, \Pi+\Delta \Pi\right)-q\left(\theta_{0}, \Pi\right)\right| \leq \mid q\left(\theta_{0}+\Delta \theta_{0}, \Pi+\Delta \Pi\right) \\
& \quad-q\left(\theta_{0}+\Delta \theta_{0}, \Pi\right)|+| q\left(\theta_{0}+\Delta \theta_{0}, \Pi\right)-q\left(\theta_{0}, \Pi\right) \mid
\end{aligned}
$$

where the triangle inequality was utilized. After utilizing the mean value theorem (Khalil, 2002), the terms on the right-hand side of (C.2) can be written as follows

$$
\begin{aligned}
& q\left(\theta_{0}+\Delta \theta_{0}, \Pi+\Delta \Pi\right)-q\left(\theta_{0}+\Delta \theta_{0}, \Pi\right) \\
& \quad=\left.\frac{\partial q\left(\theta_{0}+\Delta \theta_{0}, v_{1}\right)}{\partial v_{1}}\right|_{v_{1}=\psi_{1}}(\Pi+\Delta \Pi-\Pi)
\end{aligned}
$$

where $\psi_{1} \in[\Pi, \Pi+\Delta \Pi]$ and can be chosen as $\psi_{1}=\Pi+\Delta \Pi-$ $\rho_{1}(\Pi+\Delta \Pi-\Pi)$ with $\rho_{1} \in[0,1]$ and
$q\left(\theta_{0}+\Delta \theta_{0}, \Pi\right)-q\left(\theta_{0}, \Pi\right)$

$=\left.\frac{\partial q\left(v_{2}, \Pi\right)}{\partial v_{2}}\right|_{v_{2}=\psi_{2}}\left(\theta_{0}+\Delta \theta_{0}-\theta_{0}\right)$

where $\psi_{2} \in\left[\theta_{0}, \theta_{0}+\Delta \theta_{0}\right]$ and can be chosen as $\psi_{2}=\theta_{0}+\Delta \theta_{0}-$ $\rho_{2}\left(\theta_{0}+\Delta \theta_{0}-\theta_{0}\right)$ with $\rho_{2} \in[0,1]$. From (11)-(14), it can be seen that $q(\cdot)$ is differentiable with respect to its arguments. Also, since the measurable position signals are assumed to be bounded (see Remark 2), we can utilize (C.2)-(C.4) to obtain the following expression

$\left|q\left(\theta_{0}+\Delta \theta_{0}, \Pi+\Delta \Pi\right)-q\left(\theta_{0}, \Pi\right)\right| \leq L_{2}\left(\|\Delta \Pi\|+\left\|\Delta \theta_{0}\right\|\right) \quad$ (C.5) where $L_{2} \in \mathbb{R}$ is a positive constant. The expression given in (C.5) is same as the expression given in (16) in Assumption 5.

Similar argument can be given to show the validity of Assumption 4. To facilitate the argument, we define $t_{\Delta} \in \mathbb{R}$ as $t_{1} \leq t_{\Delta} \leq t_{2}$. After utilizing the mean value theorem, the following expression can be obtained

$\Pi\left(t_{2}\right)-\Pi\left(t_{1}\right)=\dot{\Pi}\left(t_{\Delta}\right)\left(t_{2}-t_{1}\right)$.

The left-hand side of (C.6) can be upper bounded as follows

$\left\|\Pi\left(t_{2}\right)-\Pi\left(t_{1}\right)\right\| \leq\left\|\dot{\Pi}\left(t_{\Delta}\right)\right\|\left|\left(t_{2}-t_{1}\right)\right|$.

Since the position and velocity of the moving platform are assumed to be bounded then $\dot{\Pi}\left(t_{\Delta}\right)$ is bounded; hence, (C.7) can be written as follows

$\left\|\Pi\left(t_{1}\right)-\Pi\left(t_{2}\right)\right\| \leq L_{1}\left|t_{1}-t_{2}\right|$

where $L_{1} \in \mathbb{R}$ is a positive constant. It can be seen that (C.8) is the same expression as given in (15) in Assumption 4.

\section{References}

Annaswamy, A. M., Skantze, F. P., \& Loh, A. P. (1998). Adaptive control of continuous time systems with convex/concave parameterization. Automatica, 34(1), 33-49.

Baker, S., \& Nayar, S. (1999). A theory of single-viewpoint catadioptric image formation. International Journal of Computer Vision, 35(2), 175-196.

Barreto, J. P., \& Araujo, H. (2005). Geometric properties of central catadioptric line images and their application in calibration. IEEE Transactions on Pattern Analysis and Machine Intelligence, 27(8), 1327-1333.

Boskovic, J. D. (1996). Some remarks on adaptive neuro-fuzzy systems. International Journal on Adaptive Control Signal Processing, 10, 79-83.

Boyd, S., \& Vandenberghe, L. (2004). Convex optimization. Cambridge, UK: Cambridge University Press.

Cao, C., Annaswamy, A. M., \& Kojic, A. (2003). Parameter convergence in nonlinearly parameterized systems. IEEE Transactions on Automatic Control, 48(3), 397-412.

Chen, X., \& Kano, H. (2002). A new state observer for perspective systems. IEEE Transactions on Automatic Control, 47(4), 658-663.

Chen, X., \& Kano, H. (2004). State observer for a class of nonlinear systems and its application to machine vision. IEEE Transactions on Automatic Control, 49(11), 2085-2091.

Chiuso, A., Favaro, P., Jin, H., \& Soatto, S. (2002). Structure from motion casually integrated over time. IEEE Transactions on Pattern Analysis and Machine Intelligence, 24(4), 523-535.

Dixon, W. E., Fang, Y., Dawson, D. M., \& Flynn, T. J. (2003). Range identification for perspective vision systems. IEEE Transactions on Automatic Control, 48(12), 2232-2238.

Fomin, V., Fradkov, A., \& Yakubovich, V. (1981). Adaptive control of dynamical systems. Moscow, Russia: Nauka.

Geyer, C., \& Daniilidis, K. (2000). A unifying theory for central panoramic systems and practical implementations. In Proc. European conf. computer vision (pp. 445-461) Dublin, Ireland.

Geyer, C., \& Daniilidis, K. (2002). Paracatadioptric camera calibration. IEEE Transactions on Pattern Analysis and Machine Intelligence, 24(5), 687-695.

Gupta, S., Aiken, D., Hu, G., \& Dixon, W. E. (2006). Lyapunov-based range and motion identification for a nonaffine perspective dynamic system. In Proc. American control conf. (pp. 4471-4476) Minneapolis, MN

Hu, G., Aiken, D., Gupta, S., \& Dixon, W. (2008). Lyapunov-based range identification for paracatadioptric systems. IEEE Transactions on Automatic Control, 53(7), $1775-1781$.

Ioannou, P. A., \& Sun, J. (1996). Robust adaptive control. Upper Saddle River, NJ: Prentice Hall.

Jankovic, M., \& Ghosh, B. K. (1995). Visually guided ranging from observations of points, lines and curves via an identifier based nonlinear observer. Systems and Control Letters, 25, 63-73.

Kano, H., Ghosh, B. K., \& Kanai, H. (2001). Single camera based motion and shape estimation using extended Kalman filtering. Mathematical and Computer Modelling, 34(5), 511-525.

Karagiannis, D., \& Astolfi, A. (2005). A new solution to the problem of range identification in perspective vision systems. IEEE Transactions on Automatic Control, 50(12), 2074-2077.

Khalil, H. K. (2002). Nonlinear systems (3rd ed.). New York, NY: Prentice Hall. 
Ma, L., Chen, Y., \& Moore, K. L. (2004). Range identification for perspective dynamic system with single homogeneous observation. In Proc. IEEE int. conf. robot. autom. (pp. 5207-5211) New Orleans, LA.

Ma, L., Chen, Y., \& Moore, K. L. (2005). Range identification for perspective dynamic systems with 3D imaging surfaces. In Proc. American control conf. (pp. 3671-3675), Portland, OR.

Matthies, L., Kanade, T., \& Szeliski, R. (1989). Kalman filter-based algorithms for estimating depth from image sequences. International Journal of Computer Vision, 3(3), 209-238.

Nath, N., Braganza, D., \& Dawson, D. M. (2008a). Position based structure from motion using a moving calibrated camera. In Proc. American control conf. (pp. 1764-1769), Seattle, WA

Nath, N., Braganza, D., Dawson, D. M., \& Burg, T. (2008b). Range identification for perspective vision systems: a position based approach. Technical report CU/CRB/7/18/08/1. Clemson University CRB.

Nath, N., Tatlicioglu, E., \& Dawson, D. M. (2009a). Range identification for nonlinear parameterizable paracatadioptric systems. In Proc. IEEE int. conf. systems, man, and cybernetics (pp. 4554-4559), San Antonio, TX.

Nath, N., Tatlicioglu, E., \& Dawson, D. M. (2009b). Range identification for nonlinear parameterizable paracatadioptric systems. Technical Report CU/CRB/3/31/09/1. Clemson University CRB.

Orghidan, R., Mouaddib, E. M., \& Salvi, J. (2005). Omnidirectional depth computation from a single image. In Proc. IEEE int. conf. robot. autom. (pp. 1222-1227), Barcelona, Spain.

Ortega, R. (1998). Adaptive control of a class of nonlinearly parameterized plants. IEEE Transactions on Automatic Control, 43(7), 930-934.

Sommerville, D. M. Y. (1958). An introduction to the Geometry of n-dimensions. New York, USA: Dover.

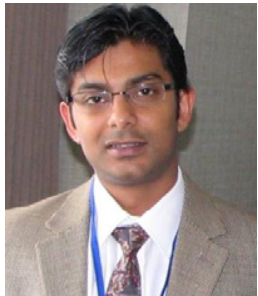

Nitendra Nath received his Bachelor of Engineering degree in Electrical Engineering, from the University of Pune, India, in 2004. He received his M.S. degree in Electrical Engineering from Clemson University in 2006. He is currently working towards his Ph.D. at Clemson University. His research interests include nonlinear control of robotic and autonomous systems, adaptive systems, estimation, neural network control, teleoperation, control/identification of vision based systems, and the development of real-time software for control applications.

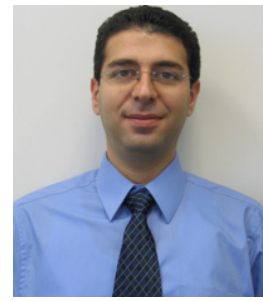

Enver Tatlicioglu received the B.Sc. degree in Electrica and Electronics Engineering from Dokuz Eylul University Izmir, Turkey, and the Ph.D. degree in Electrical and Computer Engineering from Clemson University, Clemson, SC, USA in 1999 and 2007, respectively. Upon completion of his Ph.D. degree, he worked as a post-doctoral research fellow in the Department of Electrical and Computer Engineering at Clemson University, then joined the Electrical \& Electronics Engineering Department at Izmir Institute of Technology, Izmir, Turkey where he is currently an Assistant Professor. His research interests include learning, robust and adaptive control of non-linear systems, partia state feedback and output feedback control, non-linear control techniques for kinematically redundant robot manipulators, haptic systems and teleoperation, and dynamic modelling of extensible continuum robot manipulators.

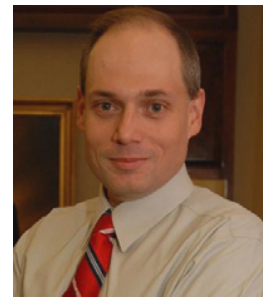

Darren M. Dawson received his B.S. degree in Electrical Engineering from the Georgia Institute of Technology in 1984. He then worked for Westinghouse as a contro engineer from 1985 to 1987 . In 1987, he returned to the Georgia Institute of Technology where he received the Ph.D. degree in Electrical Engineering in March 1990 In July 1990, he joined the Electrical and Computer Engineering Department at Clemson University where he currently holds the position of McQueen Quattlebaum Professor. From 2005 to 2007, he also served as the ECE Department Graduate Coordinator. As of August 2007, he has held the position of ECE Department Chair. His research interests include nonlinear control techniques for mechatronic systems such as electric machinery, robotic manipulator systems, overhead cranes, magnetic bearings, vision-based systems, mobile platforms (underwater vehicles, surface ships, satellites, aircraft, etc.), and mechanical friction. His interest also includes boundary control of distributed parameter systems such as paper handling/textile machines, flexible beams/robots/rotors, acoustic noise, and cable structures, robust and adaptive control of uncertain nonlinear systems, partial state feedback and output feedback control techniques and real-time hardware and software systems for contro implementation. 